information is lost and must be regenerated by indirect means, such as introducing heavy atoms into the structure. This has not yet been possible for Pf1, so a more circuitous approach has been adopted by Makowski et al., involving cycles of model building and iterative phase refinement. The stereochemical constraint of $\alpha$-helical structure and the geometrical 'box' constraint of a particle of limited radius, within which all the scattering material must be confined, combine to produce what the authors claim is a unique structural solution consistent with the X-ray intensities to spacings of about $7 \AA$.

The picture that emerges of the coat protein (see figure) is of a segmented structure. One set of $\alpha$-helical segments lies almost parallel to the particle axis at a radius of about $15 \AA$, while the other set of $\alpha$-helical segments lies at about $25 \AA$ radius and is tilted by about $25^{\circ}$ to the particle axis. The resulting tightly packed and intricately interlocked structure conforms with accepted models for packing of $\alpha$-helices and provides a stable $20 \AA$ thick protein coat around the viral DNA. Unfortunately the model is not yet sufficiently detailed for the connectivity of the segments to be established. An inner segment might connect to an outer segment lying further along the virus giving an elongated though kinked subunit, reminiscent of the single $\alpha$-helix of Marvin $\&$ Wachtel. More intriguingly the connectivity might be such that an inner segment joins to the outer segment overlying it at larger radius, giving a hairpin fold to the molecule. The latter would produce a very compact coat protein, resembling the double $\alpha$-helical hair-pin proposed as the primitive ancestor of the present day tobacco mosaic virus coat protein (McLachlan et al. J. molec. Biol. $136,203 ; 1980)$. More detailed studies are needed to choose between these possibilities but the outcome will clearly provide an important constraint on the molecular mechanisms proposed for viral morphogenesis.

\title{
Plant opportunists
}

\section{from Peter D. Moore}

ADAPTABILITY is a valuable asset for an opportunist. The maxim applies as well to the struggling weed as to the business entrepreneur, as an increasing number of studies of the genetics and population biology of plant pest species is showing. Much of the interest in this area of botany stemmed from Bradshaw's work on variation within grasses such as Argrostis tenuis (see for example, New Phytol. 59, $92 ; 1960$ ) in response to intense selective pressures operating over limited spatial distances. In particular, it was the study of heavy metal pollution in areas disturbed by mining and dumping which led him and his students to an awareness of the genetical mosaic found in the plant cover as a response to such soil stresses (Jain \& Bradshaw Heredity 21, 407; 1966). In some cases it was possible to pinpoint the precise physiological adaptation involved in the provision of selective advantages, as in the case of Snaydon and Bradshaw's work (New Phytol.60, 219; 1961) on ecotypic variation in the grass Festuca ovina on alkaline and acid soils. Here, membrane selectivity (between sodium and potasium) was shown to be closely correlated with the environmental $\mathrm{pH}$.

Complex variations and patterns in the micro-environment are frequently associated with human activity, as in the case of mine spoil, and consequently one

Peter D. Moore is in the Department of Plant Sciences, University of London King's College. might reasonably expect to find genetic diversification and intense local selection within those plant species which are especially dependent on man's activity, namely the weeds. This has indeed proved to be the case, for example, the work of Gadgil and Solbrig ( $A m$. Nat. 106, 14; 1972) on dandelions in North America, showed that these plants form a patchwork of genetically uniform populations, each of which has characters of growth, reproductive rate and seed size which could be regarded as adaptive responses to their varying local environment, such as degree of disturbance and frequency of mowing. (see Nature 252, 191; 1974, 269, 375; 1977).

But dandelions are somewhat peculiar in that they are apomictic and also can regenerate very effectively by vegetative means. Both of these characteristics lead to the conservation of a uniform genetic make-up within populations. Much interest has therefore been generated in the degree of variation and adaptation in other common weed species. An interesting series of papers concerning the genecology of a number of weeds has been published by Warwick and Briggs, who have been particularly interested in lawn plants. In the annual meadow grass, Poa annua, they found that some plants were prostrate and others erect, and that this growth habit was genetically determined (New Phytol. 81, $711 ; 1978)$. The prostrate types were better able to withstand defoliation and were well adapted to lawn conditions, which is where they were generally located. The erect forms were associated with habitats in which catastrophes were common, such as disturbed soils, and devoted a greater proportion of their energy to reproduction. The erect types can therefore be regarded as $r$-selected with respect to the prostrate types.

A subsequent analysis of populations of the broad-leaved plantain, Plantago major, has demonstrated some similarities to and some differences from the meadow grass (New Phytol. 85, 289; 1980). Populations were examined from regularly mowed lawns and from waste ground beside roads and canals. Again, there was a differentiation into populations with flattened, prostrate rosettes of leaves (found on lawns) and those with erect, tall shoots and leaves (found on roadsides). Naturally, when exposed to a régime of regular clipping at a height of $2 \mathrm{~cm}$ above ground level, the erect form suffered far more severe damage than the prostrate form. In this respect the plantains resembled the meadow grass. A consideration of Warwick and Briggs' data concerning the accumulation of vegetative and reproductive dry weight over the 14 weeks of the experiment, reveals some interesting contrasts to the meadow grass observations. Under control (unclipped) conditions, the reproductive structures (flower and fruiting spikes plus their scapes) of the lawn plantains account for between $62 \%$ and $65 \%$ of the total plant dry weight. The roadside populations, however, have a generally lower and more variable reproductive component, between $28 \%$ and $47 \%$ (based on population means). Thus, under control conditions, the prostrate form exhibits a greater degree of energy diversion to reproduction than the erect population, and this character is normally an indication of $r$-selection. Under clipped conditions, as one might reasonably predict, this difference is further exaggerated, for the shorter, less erect spikes of the lawn type are far more successful. After 14 weeks growth under a weekly mowing regime, the lawn population had a $52 \%$ to $54 \%$ investment in reproductive structures and the roadside plants were reduced to $13 \%$ to $17 \%$.

Such clear indications of ecotypic variations have not, however, been found in all the lawn weeds examined by Warwick and Briggs. In the daisy, Bellis perennis (New Phytol 85, 289; 1980), there was a great deal of variation within populations from grasslands of differing mowing and grazing histories. Transplantation into a regularly cut lawn did not necessarily diminish the reproductive vigour of some individuals from grazed habitats, but, in the case of the daisy, the importance of vegetative reproduction in the overall success of the plant is much greater than for either the plantain or the meadow grass. One cannot measure the full potential of a weed simply by its commitment to sexual reproduction. 